\section{An update on osteopathic medical education}

With this annual education issue of the JAOA, the osteopathic profession marks another year of evolution and growth.

The information contained in this annual statistical examination has been updated and revised to present a current profile of the profession and to highlight the changes that are under way. Once again, AOA staff have drawn on information compiled by the American Osteopathic Association and the American Association of Colleges of Osteopathic Medicine.

A brief glance at the data indicates that the profession continues to grow and its educational resources continue to strengthen. More students are applying for places in osteopathic medical schools, and the number of women and minority applicants is steadily growing. Freshman enrollment hit a new peak in 1988 after a brief decline, and total enrollment was fully three and a half times the enrollment in 1968. The number of graduates is once again increasing after a slight drop in 1987. Training opportunities continue to expand on the postdoctoral level, with more internship positions funded and new initiatives being taken to encourage development of residency programs.

The 1989 JAOA education issue is designed to provide a compendium of information on osteopathic medical students, osteopathic medical school finances, postdoctoral training, and continuing education. A new and valuable addition to this year's edition is an article detailing AOA research programs and their role in osteopathic medical education.

JAOA's annual education issue is intended to provide readers with a means by which to measure the profession's development and to assess ongoing changes. We hope you'll keep each year's edition handy as a reference source.

\author{
SANDRA M. WILLIAMSON \\ Executive Editor
}

\section{Medical curriculum heeds lesson in disease prevention}

Increasingly, physicians are criticized for too often dealing only with the diagnosis and treatment of a specific illness. Such criticism particularly belies the osteopathic medical philosophy: The proper approach to patient care begins with an evaluation of the total patient. Not only is the patient a body with an illness, but he or she is a person with a mind and spirit.

In that context, the environment in which a person lives, works, and plays influences an individual's health. Each of us can control that environment to some extent through behavior modification.

However, the role of behavior modification in disease prevention is practically overlooked in the traditional medical school curriculum. While such a curriculum produces physicians expert in diagnosing and treating disease, these same superbly capable physicians frequently fail to recognize the importance of preventive healthcare. With so little time in the curriculum devoted to this area, only the exceptional student develops a truly comprehensive approach to the public's healthcare needs.

In response to recent reports in the lay and medical press, which confirm the correlation between behavior modification and a decline in cardiac morbidity and mortality, the public seeks a partnership with physicians in a quest

(continued on page 1430) 